\title{
Ultrasound Assessment of Postpartum Fever
}

\author{
Kathrene Tajnert, Wayne Tie, Thomas J O’Neill, Sanja Kupesic Plavsic
}

\begin{abstract}
Postpartum fever and infection remain a significant cause of morbidity in postpartum period. Ultrasound is useful for assessment of the patients who present with temperature elevation, vaginal bleeding, pelvic and/or abdominal pain and signs of infection. Sonography can also be used to detect mastitis and breast abscess. Different types of imaging may be used in patients with refractory fever from suspected pyelonephritis, renal abscess and deep vein thrombosis. This article presents a variety of case scenarios regarding the postpartum fever. By using illustrative images the learner will be able to differentiate between the most common causes of postpartum fever, and will be able to identify typical B-mode, color Doppler, and CT images that require immediate antibiotic and/or invasive treatment.
\end{abstract}

Keywords: Postpartum fever, B-mode ultrasound, Color Doppler ultrasound, CT imaging, Endometritis, Cystitis, Pyelonephritis, Renal abscess, Deep vein thrombosis, Mastitis.

How to cite this article: Tajnert K, Tie W, O'Neill TJ , Plavsic SK. Ultrasound Assessment of Postpartum Fever. Donald School J Ultrasound Obstet Gynecol 2013;7(2):219-230.

\section{Source of support $\mathrm{Nil}$}

Conflict of interest: None declared

\section{INTRODUCTION}

Postpartum complications and specifically postpartum fever remain a major concern of pregnancies. It was found that $5.5 \%$ of all vaginal deliveries and $7.4 \%$ of cesarean deliveries eventually resulted in a postpartum infection. Overall, postpartum infection rate was found to be $6.0 \%{ }^{1}$

Postpartum fever is generally defined as a temperature greater than $38.0^{\circ} \mathrm{C}$ on any two of the first 10 days following delivery exclusive of the first 24 hours. ${ }^{2}$ When a postpartum female presents with fever, pelvic and/or lower abdominal pain, it is important to rule out diagnosis which are lifethreatening and would require urgent intervention. The differential diagnosis of postpartum complication and fever is quite broad and includes many different organ systems, such as gastrointestinal, gynecological, urological and vascular. Therefore, a cost-effective and efficient strategy, such as ultrasound, is beneficial and should be utilized.

Causes of postpartum infection can include several factors. Generally, local spread of colonized bacteria is the most common etiology for postpartum infection following vaginal delivery. Endometritis is the most common infection in the postpartum period, and accounts for $50 \%$ of all infections in patients following cesarean delivery. ${ }^{1}$ In all, it affected $3.4 \%$ of cesarean deliveries. $M$ astitis and urinary tract infections (UTIS) were the next most common causes and occurred in $5 \%$ of vaginal deliveries. Other postpartum infections include postsurgical wound infections, perineal cellulitis, respiratory complications, retained products of conception and septic pelvic phlebitis.

A detailed history of the delivery and the course of the pregnancy are vital in the evaluation of postpartum patients. Important information to inquire about includes:

- Ask about any prenatal care.

- Was the patient diagnosed or treated for any infections during the pregnancy?

- Did premature rupture of the membranes occur?

- Was the delivery vaginal or cesarean?

Patient presentation can vary widely depending on the cause and source of the complication or fever. It is important to determine if there is fever and chills, abdominal pain and foul-smelling lochia. Other more specific signs and symptoms include breast engorgement in cases of mastitis, flank pain, dysuria and frequency in UTIs. Erythema and/ or drainage from a surgical incision are present in cases of wound infections. Respiratory symptoms, such as cough, dyspnea and pleural chest pain may occur in cases of respiratory infection or pulmonary embolus $(\mathrm{PE})$.

The workup for suspected postpartum infection is often complementary when there is high evidence of suspicion. Laboratory studies are aimed at clarifying the source and etiology of the infection and should include: completeblood count, electrolytes, blood cultures, urinalysis with cultures and sensitivity tests, cervical or uterine cultures and wound cultures. Lactate can be useful if sepsis suspected. Lastly, coagulation studies are necessary when pelvic thrombosis, deep vein thrombosis (DVTs), pulmonary embolism is suspected. Here, we present case study series of the patients presenting with postpartum fever.

\section{CASE STUDIES}

\section{Case \#1}

A 23-year-old G1P1 delivered a 38-week infant by cesarean section 30 hours ago. Initially, the patient was presented to the hospital with confirmed premature rupture of membranes. A fter a prolonged labor and arrest of cervical dilation, a C-section was performed. Prenatal course consisted of a UTI for which she was treated for. She states she received no prenatal care, but did take prenatal vitamins. Two days after delivery, she complains of increasing lower 


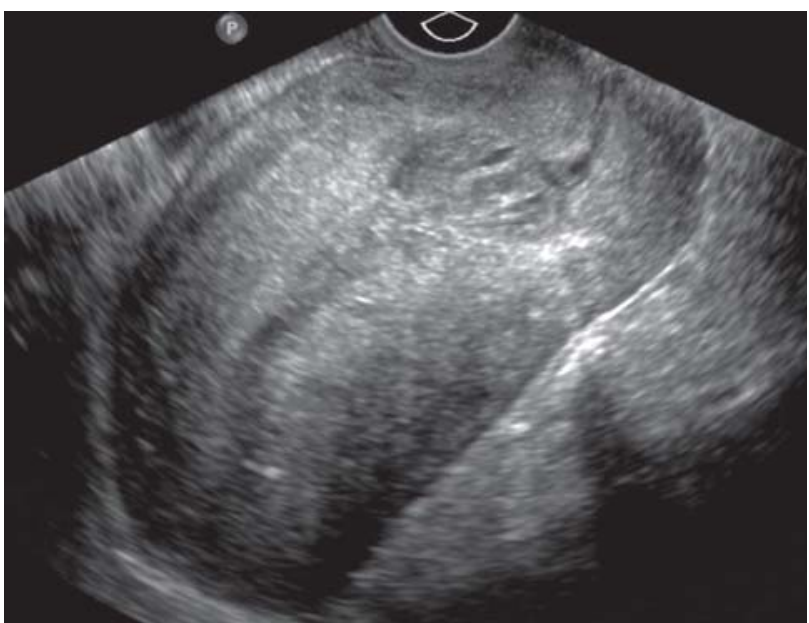

Fig. 1A: Transvaginal ultrasound of postpartum uterus. Note heterogeneous appearance of the endometrium and myometrium and distended uterine cavity filled with blood and clots

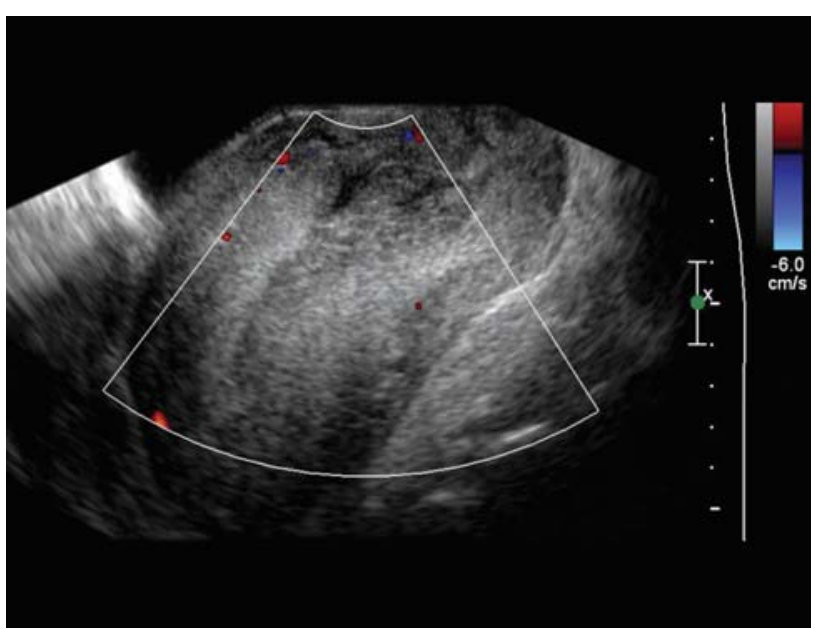

Fig. 1B: Transvaginal color Doppler image of the same patient. Absence of endometrial mass and lack of blood flow signals within the cavity reasonably excludes residual products of conception

abdominal pain, frequency and dysuria. She also describes heavy, foul smelling lochia. Her vital signs show a temperature of $98.9^{\circ} \mathrm{F}\left(37.2^{\circ} \mathrm{C}\right)$; pulse 70 ; blood pressure 100/60; and respirations 12. On examination, her lungs are clear, cardiac exam is normal, abdomen is soft, uterine fundus is firm and tender, and she reports mild suprapubic tenderness. $C B C$ reveals a W BC count of 13.1; hemoglobin $11 \mathrm{~g} / \mathrm{dl}$ and hematocrit $33 \%$. U rinalysis is positivefor RBCs, negative for WBCS, nitrites and leukocyte esterase. Urine culture is pending. Endometrial culture is obtained and pending. Pelvic ultrasound is performed and results are shown in Figures $1 \mathrm{~A}$ and $\mathrm{B}$.

\section{Case \#2}

A 25-year-old G1P1 delivered a 38-week infant by cesarean section. The patient was admitted with premature rupture of membrane. A fter a prolonged labor and arrest of cervical

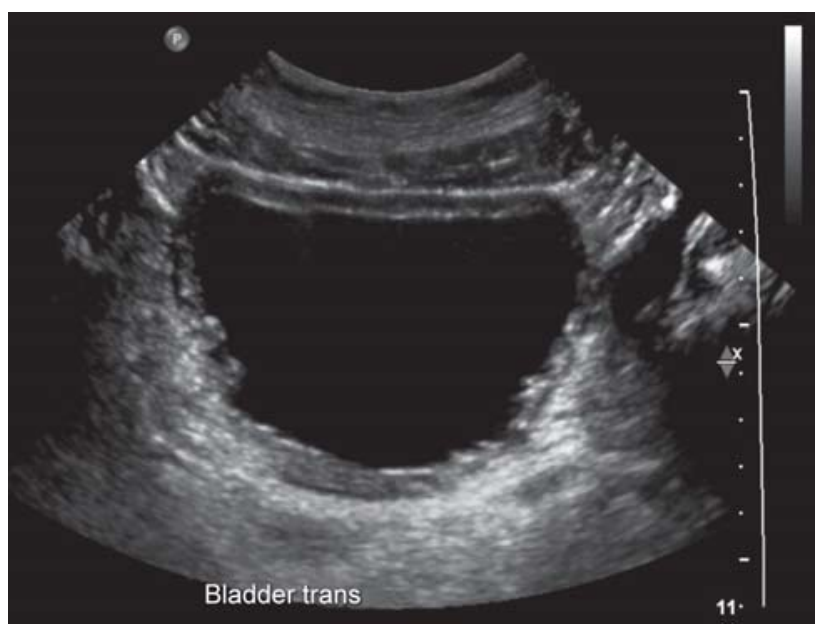

Fig. 2A: Prevoid gray scale ultrasound image of the urinary bladder

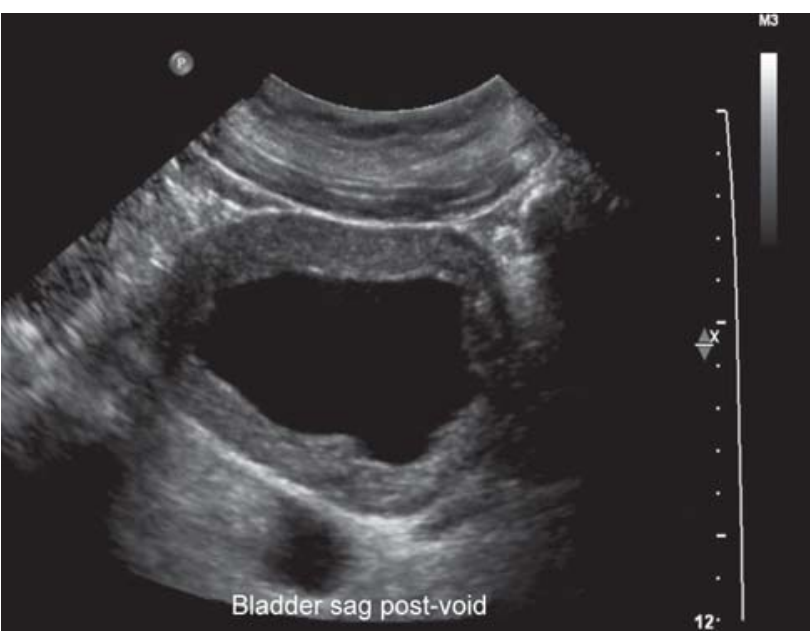

Fig. 2B: Postvoid gray scale B mode ultrasound of the urinary bladder. Note diffuse mural thickening of the urinary bladder

dilation a C-section was performed. She had an epidural, with an indwelling catheter for 24 hours. During the second trimester she was diagnosed with a UTI for which she was treated. Forty-eight hours after delivery, she complains of increasing lower abdominal pain, urgency, burning sensation when urinating, frequency, passing strong smelling urine and describes feeling of pressure in suprapubic area. $V$ ital signs show a temperature of $98.9^{\circ} \mathrm{F}$ $\left(37.2^{\circ} \mathrm{C}\right)$; pulse 70 ; blood pressure $100 / 60$; and respirations 12. On physical examination, her lungs are clear, cardiac exam is normal, abdomen is soft. Her breasts have no erythema. U terine fundus is firm and nontender, and she reports mild suprapubic tenderness. L ochia is normal. CBC reveals a WBC count of 11.1 , hemoglobin of $12 \mathrm{~g} / \mathrm{dl}$ and hematocrit $34 \%$. U rinalysis is positive for RBCs and WBCs, with positive nitrites and leukocyte esterase. U rine culture is pending. Pelvic ultrasound is preformed and results are shown in Figures $2 \mathrm{~A}$ and $\mathrm{B}$. 


\section{Case \#3}

A 32-year-old G 3P2A 1 presents to the emergency department 2 days after being discharged for an uncomplicated labor and vaginal delivery. Her postpartum course has been unremarkable and has been breastfeeding without difficulty. On presentation she complains of increasing subjective fever, chills and malaise over the last 12 hours. She also states she has been feeling mild

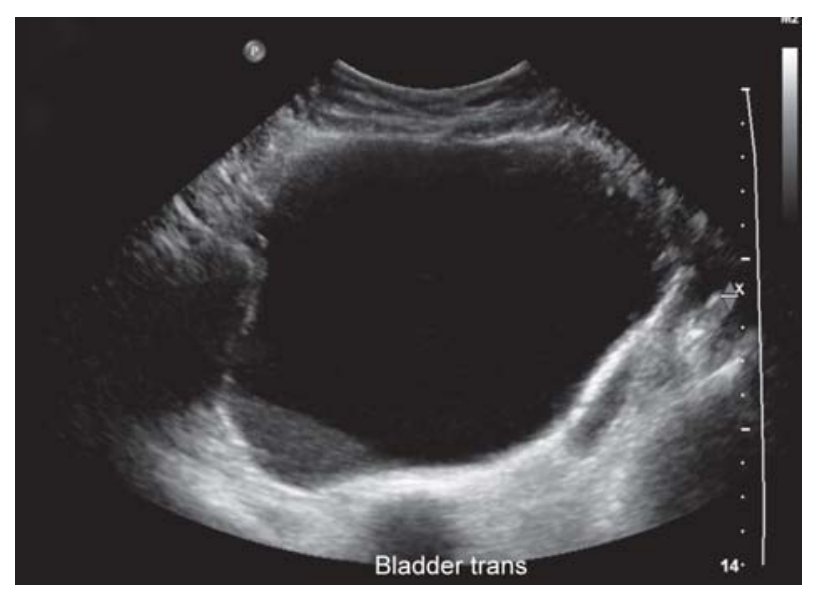

Fig. 3A: Transverse gray scale ultrasound of the urinary bladder demonstrates hypoechoic debris layering posteriorly in the urinary bladder

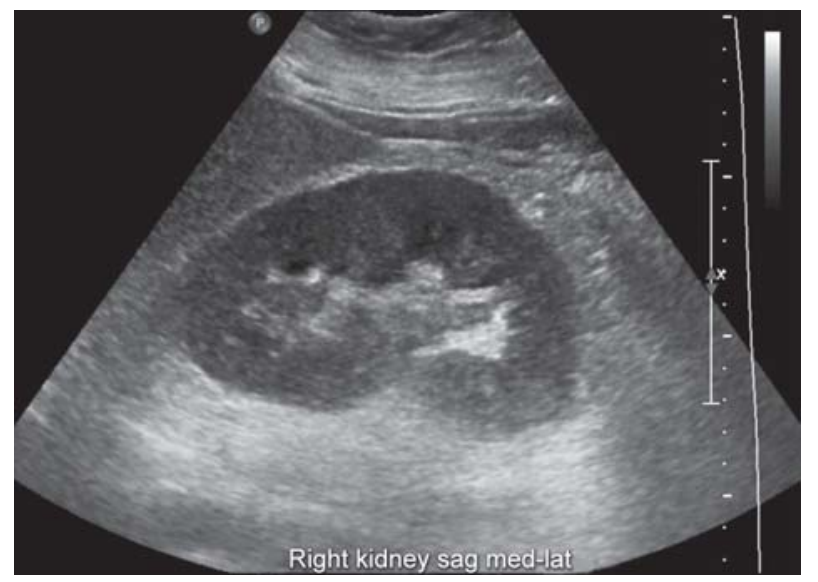

Fig. 3B: Gray scale ultrasound demonstrates mild enlargement of the right kidney with indistinct corticomedullary differentiation of the upper and lower poles of the right kidney

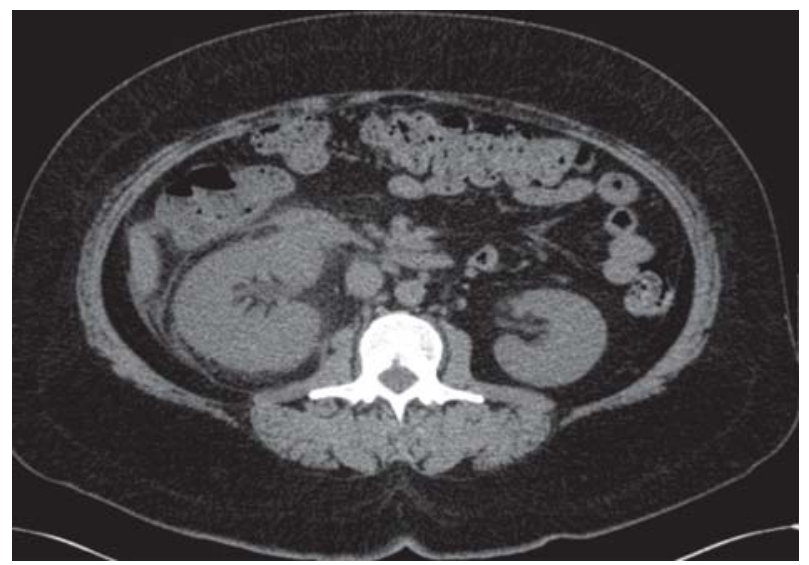

Fig. 3C: Axial CT without contrast of the same patient demonstrates an enlarged right kidney with perinephric fat stranding abdominal pain, nausea and one episode of vomiting. She states that she is having moderate bilateral flank and back pain. Her vital signs are temperature $101.6^{\circ} \mathrm{F}$, pulse 108 , blood pressure $127 / 88$, and respirations 18 . On examination, her lungs are clear, cardiac exam is normal, abdomen is soft, uterine fundus is firm and nontender, costovertebral tenderness is present. She denies suprapubic tenderness. Her WBC count is 13,800 , with predominately neutrophils. U rinalysis shows $2+$ leukocyte esterase, positive nitrite and trace blood. Microscopy shows 25 to 50 white blood cells per high-power field, 0-3 red blood cells and many bacteria. $\mathrm{Her}$ imaging results are demonstrated in Figures $3 \mathrm{~A}$ to $\mathrm{C}$.

\section{Case \#4}

A 30-year-old G2P2 comes to the hospital with 7-day history of progressively worsening right-sided flank pain with fever and chills. The patient delivered her child 4 weeks ago. Her pregnancy was complicated by gestational diabetes and she

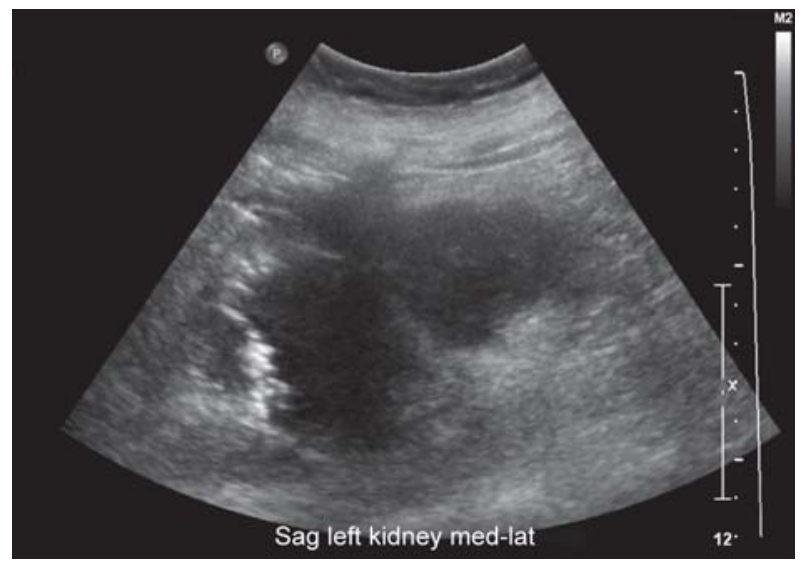

Fig. 4A: Gray scale B-mode ultrasound image of the left kidney demonstrates a hypoechoic fluid collection with low-level internal echoes

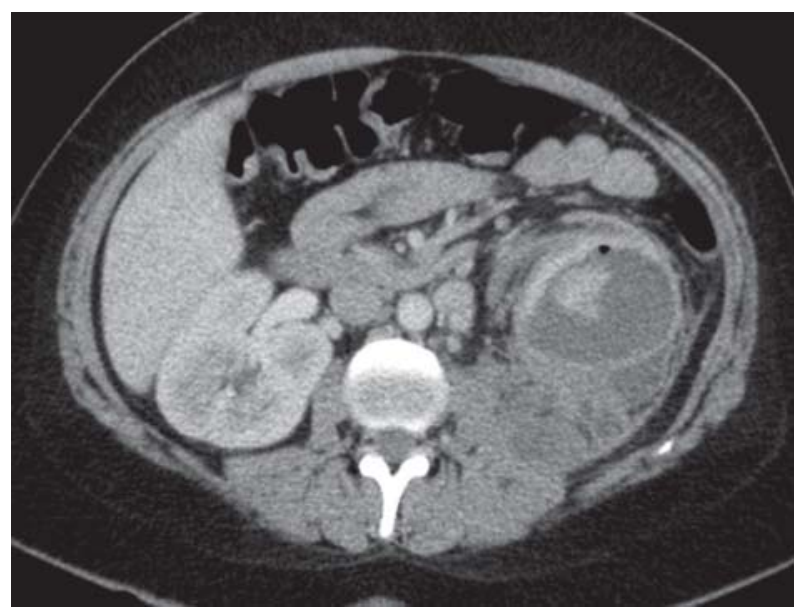

Fig. 4B: Contrast-enhanced axial CT image at the level of the kidneys demonstrates a large gas-containing intrarenal fluid collection with peripheral enhancement and perinephric fluid collection with significant perinephric fat stranding, thickening of the anterior and posterior pararenal fascia and involvement of the ipsilateral psoas muscle 
has a past medical history of recurrent UTIs. Her temperature is $39.4^{\circ} \mathrm{C}$, blood pressure $105 / 65$, pulse 100 beats per minute, and respirations 16/minute. Physical exam shows right costovertebral angle tenderness. U rinalysis shows positive nitrites and leukocyte esterase with significant bacteriuria. Patient is on day 5 of ampicillin and gentamicin without improvement. Figures $4 \mathrm{~A}$ and $\mathrm{B}$ demonstrate her kidney ultrasound and abdominal CT findings.

\section{Case \#5}

A 35-year-old G2P2 woman complains of left-sided leg edema. She delivered her child 6 days earlier by $C$-section. Six days postpartum she presents with persistent fever despite antibiotics. There is gradual onset of localized tenderness and erythema along a venous path on the left lower extremity. The patient states that she has had a history of varicose veins but denies any recent trauma or similar

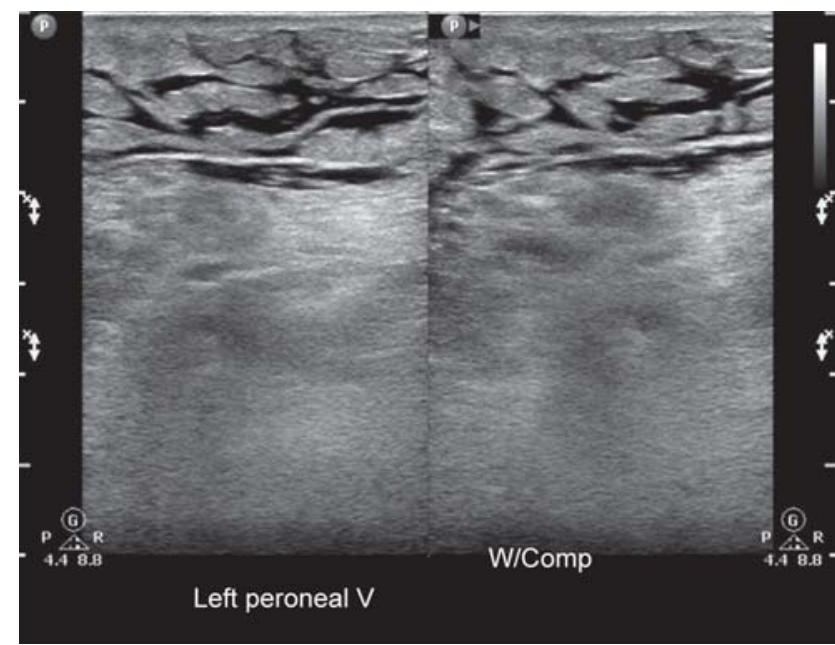

Fig. 5A: Gray scale B-mode ultrasound demonstrates anechoic fluid insinuating between the subcutaneous fat of the left lower extremity with normal compressibility of the deep veins. Ultrasound image was obtained at 36 weeks gestation

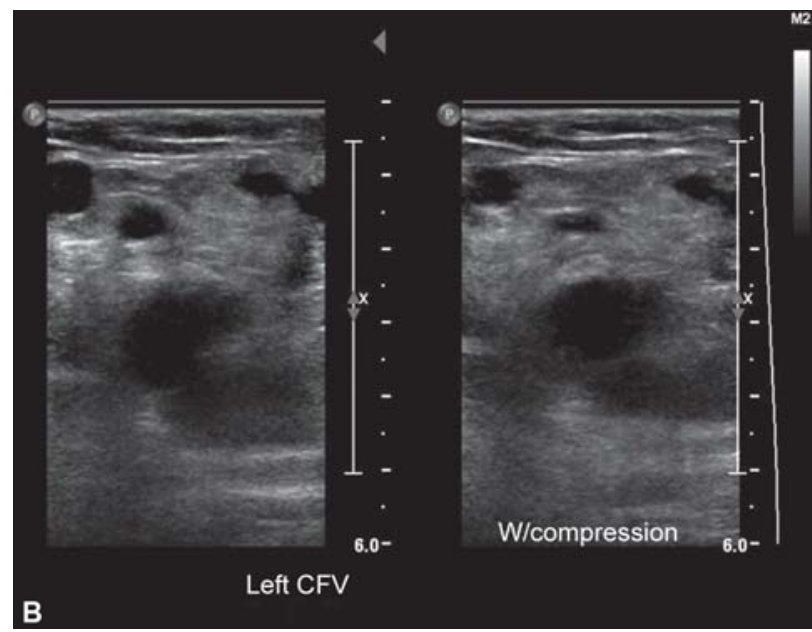

episodes involving the leg. She has an otherwise insignificant past medical history. Her temperature is $38.7^{\circ} \mathrm{C}$, blood pressure $110 / 70$, pulse 87 beats per minute. Physical exam shows tenderness to pal pation over the area of erythema and pain upon dorsiflexion of the left foot. Figures $5 \mathrm{~A}$ to $\mathrm{F}$ show B-mode, color Doppler and bone scan images.
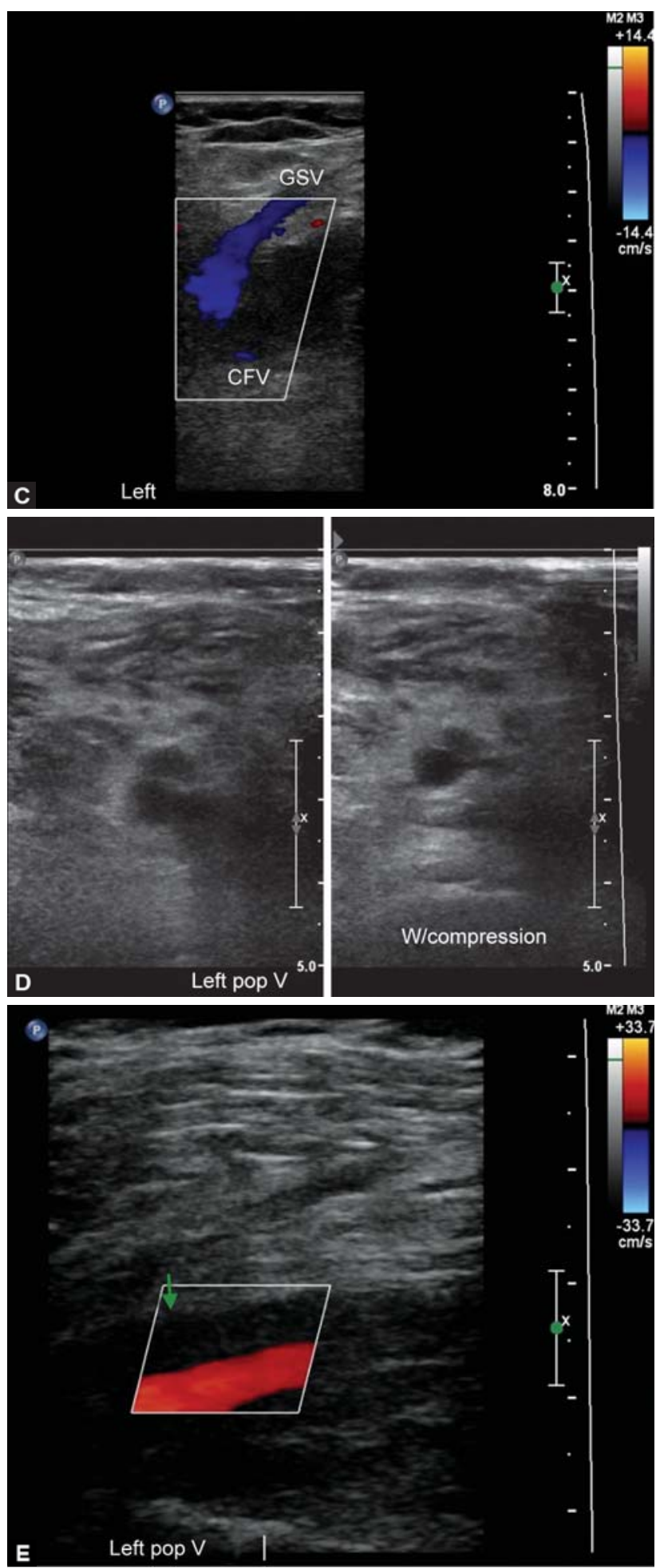

Figs 5B to E: The same patient 5 days after delivery. Gray scale ultrasound of the left lower extremity demonstrates lack of compressibility of the deep venous system extending from the common femoral vein to the popliteal vein with intraluminal echoes (Figs $5 B$ and $D$ ) and lack of flow on color flow Doppler as well as diffuse subcutaneous edema (Figs $5 \mathrm{C}$ and $\mathrm{E}$ ) 


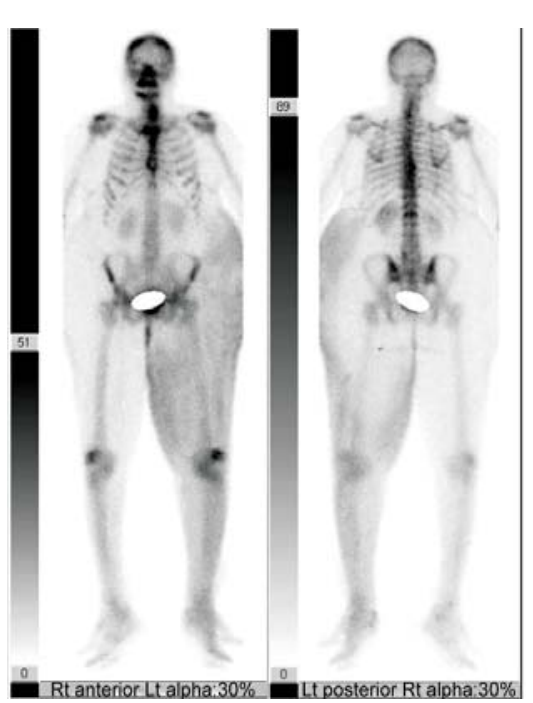

Fig. 5F: The same patient as on previous ultrasound images. Whole body anterior and posterior planar imaging after injection of Tc99m-MDP (bone scan) demonstrates diffuse radiotracer accumulation in the soft tissues of the left lower extremity extending to the left flank

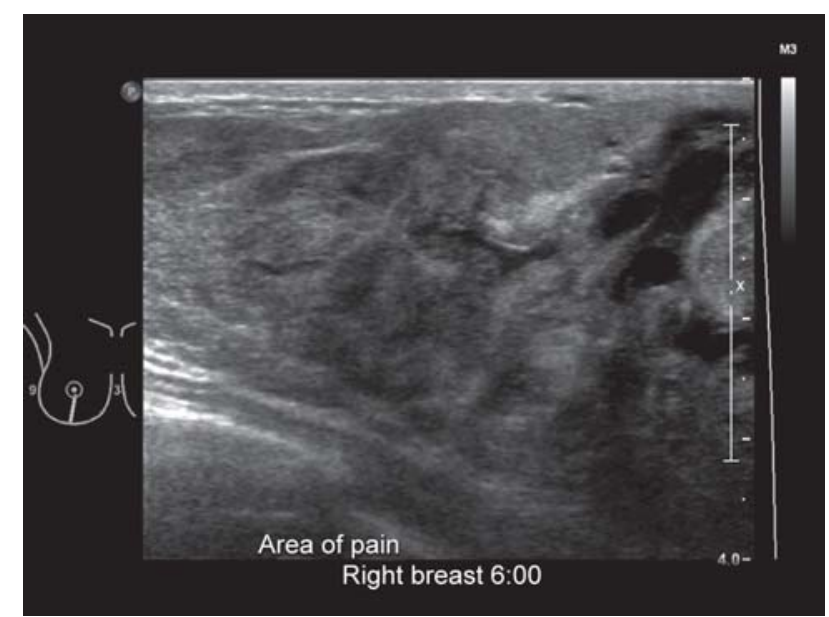

Fig. 6A: Gray scale ultrasound at 6 o'clock in the right breast

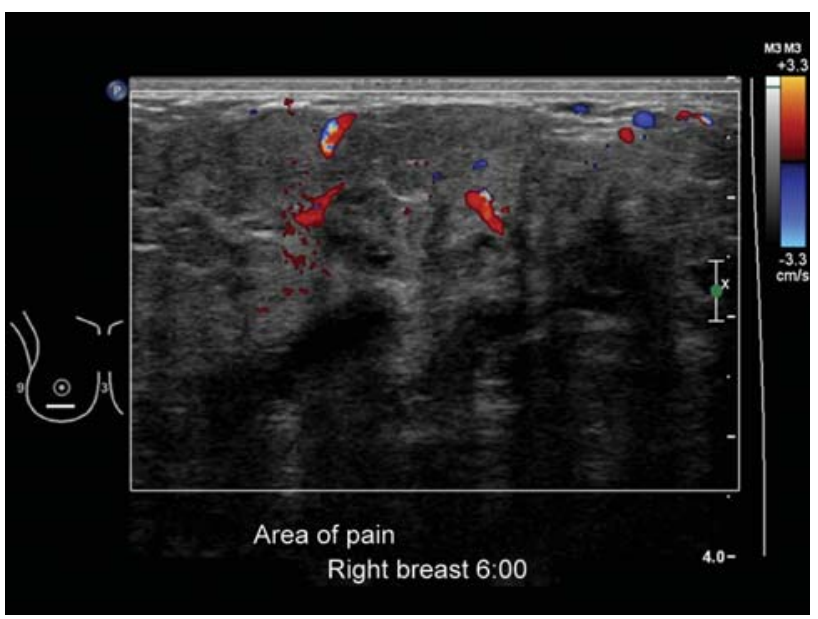

Fig. 6B: Color flow Doppler at 6 o'clock in the right breast demonstrates diffuse skin thickening with ill-defined edematous breast tissue without defined mass or fluid collection

\section{Case \#6}

A 23-year-old G1P1 delivered her first baby 12 days ago after an uncomplicated labor and vaginal delivery. Currently she describes moderate tenderness to her right breast. She has continued the breastfeeding attempts and is currently working with a lactation specialist. Prior to discharge, her temperature is $38^{\circ} \mathrm{C}$; blood pressure, pulse and respirations are all within normal limits. On physical exam, her right breast is firm, erythematous and diffusely tender. U terine fundus is appropriate and nontender. Figures $6 A$ and $B$ illustrate her B-mode and color Doppler left breast ultrasounds.

\section{Case \#7}

A 29-year-old G2P2 presents 4 weeks postpartum with complaints of fever, chills, deep breast pain and severe fatigue. She had an uncomplicated labor and vaginal

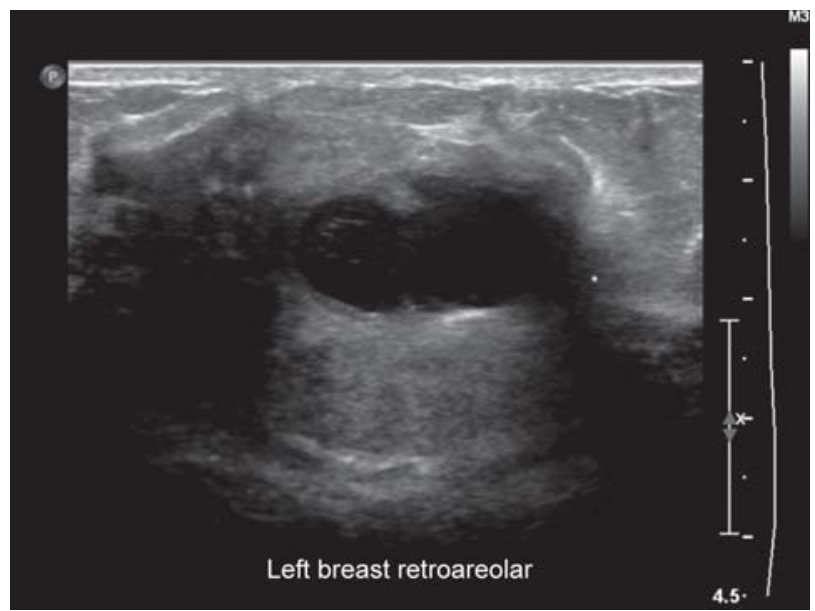

Fig. 7A: Gray scale B-mode ultrasound imaging of the left breast

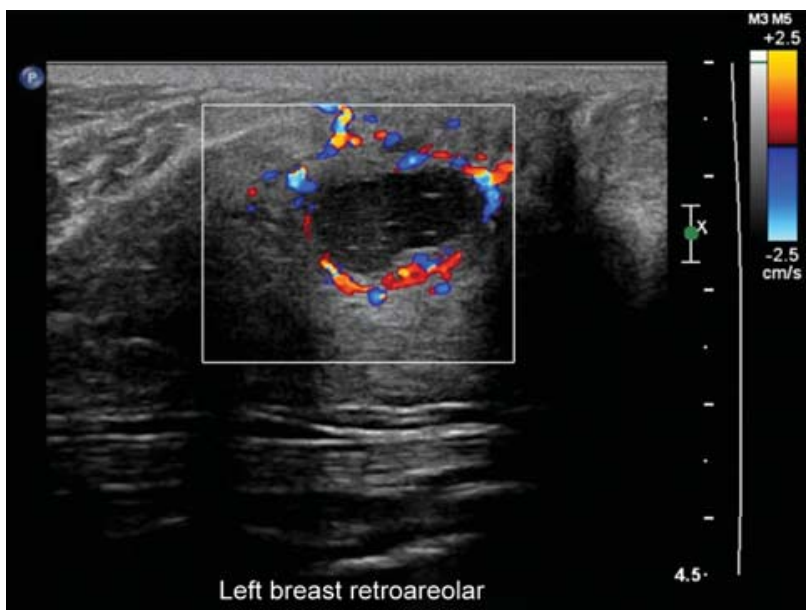

Fig. 7B: Color-flow Doppler imaging of the left breast lesion. Note an ovoid parallel retroareolar hypoechoic complex cystic mass with posterior acoustic enhancement, a slightly irregular thick wall and increased flow peripherally on color flow Doppler 
delivery. She states that she had difficulty in breastfeeding and describes problems with left breast engorgement and cracked nipples. She describes that she has never had symptoms with this severity in the past. Initially, she assumed her symptoms were due to breast engorgement and therefore was reluctant to seek immediate treatment. It has now been 1 week since the onset and presence of a pal pable mass, sw elling, redness, warmth and pain of the left breast. Her vital signs are temperature $39.5^{\circ} \mathrm{C}$, pulse 88 , blood pressure $120 / 80$ and respiratory rate 20 . On physical examination, her lungs are clear, cardiac exam is normal; abdomen is soft and nontender. On breast examination the left breast exhibits periareolar erythema, warmth, edema and an extremely tender fluctuant mass. M oderate left axillary lymphadenopathy is present. Discharge is noted with palpation of the nipple. Ultrasound of the breast is performed and results are shown in Figures 7A and $B$.

\section{DISCUSSION}

\section{Case \#1: Endometritis}

Endometritis is a polymicrobial infection of the decidua or endometrium, which can expand to the myometrium and other surrounding tissues. It occurs in about 1 to $3 \%$ of births, and is up to 10 times more common after cesarean section. ${ }^{3}$ Endometritis is often classified as acute or chronic based on histological appearance. A cute endometritis is the most common cause of fever in the postpartum period. L ocal spread of colonized bacteria and ascending infection are common etiologies for postpartum infection. The route of delivery is the most important risk factor for developing postpartum endometritis. Endometritis is the most common complication associated with cesarean delivery, and its incidence varies from 5 to $85 \%$ depending on the patient populations surveyed. ${ }^{4} \mathrm{~A}$ Iso, the prolonged labor, premature membrane rupture, prolonged membrane rupture and multiple cervical exams are other risk factors. The pathogenesis of uterine infection after cesarean delivery can include several factors. These include an infected surgical incision, local spread of bacteria which colonized the cervix and vagina gain access to the uterus during labor and surgery. M ost bacteria involved in endometritis have low virulence and rarely cause infection in healthy tissues. ${ }^{5} \mathrm{~T}$ he microorganisms most likely causing her symptoms are: Streptococcus, E. coli, Mycoplasma and U reaplasma.

Postpartum endometritis is most often a clinical diagnosis based on the presentation of postpartum fever. A fever of less than $38.3^{\circ} \mathrm{C}$ in the first 24 hours after delivery usually resolves. Endometritis should be suspected when a temperature of more than $38^{\circ} \mathrm{C}\left(100.4^{\circ} \mathrm{F}\right)$ recorded twice after the first 24 hours postpartum, or a fever of more than $38.3^{\circ} \mathrm{C}\left(101.0^{\circ} \mathrm{F}\right)$ at any time. ${ }^{6}$ In addition to fever, tachycardia, lower abdominal pain, uterine tenderness and foul-smelling lochia are often present. ${ }^{4}$ Besides endometritis, other causes should be considered in the differential in this patient. The presence of fever with flank pain, dysuria and frequency of UTIs would suggest pyelonephritis. If erythema and drainage from the surgical incision or episiotomy site are evident, suspect postsurgical wound infection. Also if she presented with respiratory symptoms, such as cough, pleuritic chest pain, or dyspnea, consider respiratory infection or atelectasis. Lower UTIS usually do not cause significant temperature increase but if high fever and chills are present consider renal abscess or breast abscess. If the patient does not respond to antibiotics the differential is great and includes collagen vascular disease, venous thromboembolism, drugs fever and septic pelvic thrombophlebitis. ${ }^{4}$

The initial evaluation of a patient with suspected endometritis is necessary to perform a complete blood count, complete metabolic panel, blood cultures, if sepsis is suspected, urinalysis, with cultures and sensitivity tests. Cervical and/or endometrial culture is also needed. A transvaginal ultrasound can help in confirming the diagnosis. As shown in Figure $1 \mathrm{~A}$ the transvaginal ultrasound of postpartum endometritis demonstrates heterogeneous appearance of the endometrium and myometrium and distended uterine cavity filled with blood and clots indicating endometritis. A transvaginal color Doppler in Figure 1B shows a lack of perfusion within the cavity, which ultimately rules out residual products of conception as the cause of the clinical symptoms.

Once diagnosis is confirmed, parental antibiotic should be started. Initial antimicrobial treatment is a combination of an aminoglycoside and clindamycin. Studies demonstrate similar efficacy in once daily dosing and in dosing every 8 hours. ${ }^{3,7}$ A Iternatively, an aminoglycoside plus metronidazole with or without ampicillin may also be used. M ild cases of endometritis after vaginal delivery can be treated with oral antimicrobial agents (e.g. doxycycline, clindamycin). Moderate-to-severe cases, including those involving cesarean deliveries, should be treated with parenteral broad-spectrum antimicrobials.

\section{Case \#2: Cystitis}

UTI is a common complication during pregnancy and in the postpartum period. ${ }^{8} \mathrm{M}$ ost UTIs are classified by three separate entities. These include acute uncomplicated cystitis, describing a lower tract infection, acute uncomplicated pyelonephritis, describing an upper tract infection, or complicated UTI which is commonly associated with urinary 
catheterization or instrumentation, or anatomic or functional abnormalities. This case describes a presentati on of cystitis, which is the inflammation of the lower urinary tract. Cystitis typically presents with fewer or no systemic signs as compared to acute pyelonephritis. A s with other postpartum infections, the cause of a UTI in postpartum can be endogenous, caused by the normal vaginal or skin flora reaching a sterile environment such as the bladder. The source can also be exogenous caused by contamination. Postpartum bacteriuria occurs in 3 to $34 \%$ of patients, resulting in a symptomatic infection in approximately $2 \%$ of these patients. ${ }^{9}$ Risk factors for postpartum cystitis are cesarean delivery, forceps delivery, vacuum delivery, maternal renal disease, pre-eclampsia, epidural anesthesia, bladder catheterization, length of hospital stay, and previous UTI during pregnancy. The risk of postpartum infection is close to a 5-fold increase after $\mathrm{C}$-section compared with vaginal birth. ${ }^{10}$ Although, the risk of UTI in vaginal vs cesarean section is still debated, according to a study by Leth et al, the prevalence of postpartum UTI was $2.8 \%$, after CS and $1.5 \%$ after vaginal birth. ${ }^{10}$ However, other studies find that vaginal birth increases the risk of UTI more so than delivery by $\mathrm{C}$-section. The discrepancies are thought to vary based on use of urinary catheters during vaginal delivery and a consequence of use of epidural anesthesia. ${ }^{10}$

The most common pathogen of postpartum (is this supposed to be UTI?) is E. coli. In pregnancy, group B streptococci are a major pathogen. Other causative organisms include Staphylococcus saprophyticus, E. faecalis, Proteus and K. pneumoniae. Signs and symptoms include pressure in the lower pelvis, dysuria, polyuria or urinary urgency. A Iso hematuria, dark and cloudy, strongsmelling urine may be apparent. On exam, there is suprapubic tenderness. L ow-grade fever is often a primary early sign of postpartum cystitis. ${ }^{11}$ Other differential diagnoses to consider in this patient are pyelonephritis (with presence of flank pain, dysuria and frequency), endometritis (with history of abnormal lochia and/or vaginal bleeding) and postsurgical wound infection (with erythema and drainage from the surgical incision or episiotomy site).

The evaluation of a patient with suspected cystitis should include a complete blood count, complete metabolic panel, blood cultures, if sepsis is suspected, urinalysis, with cultures and sensitivity tests, and perform a pelvic ultrasound (Figs 2A and B). On ultrasound, pre- and postvoid gray scale images often demonstrate diffuse mural thickening of the urinary bladder suggesting cystitis. M obile or layering intraluminal echogenic material, though not specific, may also represent cellular debris resulting from infectious cystitis.
Treatment for cystitis should be prompt to prevent further infection and complications. The 2010 Infectious Disease Society of A merica guidelines for uncomplicated cystitis in nonpregnant patients recommend nitrofurantoin (Macrobid, $100 \mathrm{mg}$ orally twice daily for 5-7 days). A alternative treatment is 3 days of trimethoprimsulfamethoxazole (TMP-SMX).

\section{Case \#3: UTI and Acute Pyelonephritis}

This case demonstrates a classic presentation of pyelonephritis. Pyelonephritis is an upper UTI most likely affecting the kidneys and/or the ureters. The incidence of this infection in the postpartum period is comparable to the time of pregnancy, although several risk factors can alter the risk in the postpartum period. ${ }^{12}$ Studies have determined that there is a 5 to $10 \%$ prevalence of asymptomatic bacteriuria in pregnancy. Up to $25 \%$ of asymptomatic patients will progress to symptomatic infection. ${ }^{13}$ Postpartum surveillance of bacteriuria in the postpartum period detected the prevalence of asymptomatic bacteriuria in $34.5 \%$ of patients. ${ }^{14}$ Due to the high prevalence of postpartum bacteriuria there is also a high potential to progress to pyelonephritis. The most significant percentage of acute pyelonephritis occurs in the first 3 weeks postpartum. ${ }^{15}$ Several factors increase the risk of developing a postpartum UTI and potential for progression to pyelonephritis. This includes urine stasis during pregnancy which carries on the early postpartum period, perineal discomfort in postpartum period due to tears, episiotomy or other various injuries, bladder insensitivity to increased urinary tension in immediate postpartum period and catheterization. The bladder is originally inoculated with an infectious organism, which then migrates up the ureter to the central collecting system. The endotoxins are believed to inhibit ureteral peristalsis by blocking the $\alpha$-adrenergic nerves within smooth muscle, thus creating a functional obstruction. The obstruction compromises the forward flow of urine, which is a normal protective mechanism against upper UTI. Bacteria enter the renal tubules at the papillary tip and cause an inflammatory response that extends up the tubule and into the renal interstitium. Severe complications of pyelonephritis are potential renal failure, septic shock, renal abscess and renal vein thrombosis.

Pyelonephritis is diagnosed by presence of clinical symptoms, urinary findings and imaging. Therefore, workup for UTI should include urine analysis (dipstick) and urine culture with sensitivities. Results of urine analysis most commonly have positive results for nitrites which signify presence of Gram-negative bacteria, leukocytes esterase which point to infection, WBCs, RBCs and protein. A s in 
the patient presented, a pyelonephritis is highly suggested based on her signs and symptoms (fever, costovertebral angle tenderness, nausea, abdominal pain) and presence of pyuria and a positive urine culture $\geq 100,000$ colonies. $^{16}$ Pyuria is defined by greater than 5 to 10 W BCs per highpower field (hpf). Other evidence of upper tract disease on blood test includes leukocytosis with a neutrophil shift on blood test, elevated erythrocyte sedimentation rate, elevated C-reactive protein levels, and occasionally positive blood cultures that grow the same organism as cultured from the urine. Figures $3 \mathrm{~A}$ to $\mathrm{C}$ illustrate ultrasound and abdominal $\mathrm{CT}$ findings of the patient presenting with pyelonephritis. Contrast-enhanced CT is the imaging study of choice for detection of acute pyelonephritis, and has a greater sensitivity as compared to ultrasound. ${ }^{17}$ Common CT findings are poorly defined wedge-shaped lesions that have decreased attenuation. Often this radiates from the papilla in the medulla to the cortical surface. Also, bands of hyperattenuation and hypoattenuation oriented in parallel of the tubules and collecting ducts can be seen. ${ }^{17}$

Treatment of pyelonephritis includes initial empirical therapy, which should be started while awaiting a urine culture and susceptibility test and should be tailored based on the microorganism. ${ }^{18}$ In general, oral ciprofloxacin $500 \mathrm{mg}$ twice daily for 7 days is the choice of antibiotic for patients not requiring hospitalization. If an initial one-time intravenous agent is used, $1 \mathrm{gm}$ of ceftriaxone could be used instead of fluoroquinolone. ${ }^{18}$ If patient is considerably ill and hospitalization is necessary, she should initially be treated with intravenous antibiotics. These include fluoroquinolones, aminoglycosides or extended-spectrum cephalosporins or extended-spectrum penicillins. The ultimate choice of the antibiotic needs to be specific to urine culture and sensitivities, and the patient's presentation. ${ }^{18}$

\section{Case \#4: Renal Abscess}

Renal abscess is a complication of pyelonephritis, seen in about 1 to 10 cases per 10,000 patients. ${ }^{19}$ The clinical presentation of this patient is continuous high fever and chills, with progressive costovertebral tenderness unrelieved by antibiotics. A s with all cases of acute urinary symptoms, urinalysis and comprehensive metabolic panel should be the first steps in diagnosis, followed by urine and blood cultures. U rinalysis may reveal presence of leukocytes and bacteria while also exploring differential diagnoses such as glomerular and tubular disease in the presence of genitourinary symptoms. The definitive approach to complicated UTI is imaging, including B-mode/color Doppler ultrasound and CT scan of the abdomen and pelvis (Figs $4 \mathrm{~A}$ and $\mathrm{B}$ ). Imaging may reveal potential obstruction or anatomical problems of the urinary tract especially in the presence of abscess formation.

Perirenal abscess on B-mode ultrasound will typically demonstrate a round pocket of hypoechogenicity surrounded by a thin rim of enhancement. ${ }^{20}$ Figure $4 \mathrm{~A}$ shows a gray scale B-mode ultrasound demonstrating hypoechoic fluid collection within the kidney and areas of low-intensity internal echoes. A dditionally, renal abscesses may present with dilation of the renal pelvises and calyces due to obstruction. ${ }^{19}$ Color Doppler ultrasound often shows increased peripheral vascularity with areas of perfusion defects that represent pockets of nephritis. ${ }^{20}$ Ultrasound provides a good initial tool to rule in or rule out possible abscess; however, CT scan of the abdomen and pelvis prevails as the most definitive imaging device in presence of a hypoechoic perinephric mass on ultrasound. ${ }^{21} \mathrm{CT}$ scan helps in differentiating between renal cysts and renal carcinoma under positive ultrasound findings. It is also hel pful in determining further complications such as spread of infection into the retroperitoneum, bowels or even pancreas. ${ }^{21}$ In this patient's case of isolated renal abscess, treatment is percutaneous drainage and prolonged course of antibiotics with follow-up with serial ultrasound to monitor resolution of abscess.

\section{Case \#5: Deep Vein Thrombosis}

DV Ts are a major complication of pregnancy that occurs in 0.5 to 2.2 women per 1,000 pregnancies. ${ }^{22}$ Typically, DVTs occur more than 5 to 6 days after surgery or prolonged periods of stasis. Pregnancy is a state of heightened risk for developing thromboemboli due to the static nature of the patient, the hyperestrogenic state of pregnancy, and venous distention causing damage to the vasculature. ${ }^{23}$ During pregnancy, there is an increase in procoagulant factors and a reduction in fibrinolytic factors. Fibrinogen increases after the third month of pregnancy as well as factors VII, VIII, $\mathrm{IX}$ and $\mathrm{X}$ with a simultaneous decrease in protein $\mathrm{S} .{ }^{24} \mathrm{In}$ this patient, there are overt physical signs of venous involvement in the lower leg with fever and positive Homan's sign. With a strong clinical suspicion for DVT, ultrasonography of the lower leg vasculature is first line in diagnosis.

While contrast venography is the gold standard in detecting thromboemboli, compression ultrasonography serves as a noninvasive alternative that has a sensitivity of $97 \%$ and specificity of over $98 \%$ in detection of proximal DV Ts and 70\% sensitivity in calf vein DV Ts. ${ }^{25}$ Fortunately, distal DVTs rarely progress to proximal DVTs and/or pulmonary emboli. ${ }^{25}$ Combining sonography with $D$-dimer levels and serial imaging can increase the effectiveness of 
ultrasound in distal thrombosis. ${ }^{22}$ Typically, as seen in Figures $5 B$ and $D$, gray scale ultrasound shows lack of compressibility of the deep veins and color Doppler reveals reduced blood flow with subcutaneous edema (Figs 5C and $\mathrm{E}$ ).

The patient currently does not have dyspnea or chest pain which lessens the suspicion of PE. W hile pregnancy is associated with development of DVT's, PE is an important complication of specifically the postpartum period. ${ }^{26}$ Epidemiologically, PE frequently occurs 4 weeks postpartum and its occurrence is about 0.45 per 1,000 births. ${ }^{27}$ Treatment for both PE and DVT includes enoxaparin and prevention of venous stasis via compression and ambulation. Recurrent PE or DVT may be indication for chronic anticoagulation.

\section{Case \#6: Mastitis}

A cute mastitis is a common cause of postpartum fever, and may be seen in 2 to $10 \%$ of breastfeeding women. ${ }^{28}$ The 23-year-old patient presents with localized breast symptoms including erythema and tenderness to palpation. Other typical symptoms include high fever, induration over the area of mastitis and swelling. M astitis may sometimes be confused with pain and cracking of the nipples which is frequently seen at the beginning of breastfeeding. M ost commonly, it is caused by $\mathrm{S}$. aureus but may also involve methicillin-resistant $\mathrm{S}$. aureus and anaerobic organisms. ${ }^{29}$ Clinical management of uncomplicated mastitis includes empiric antibiotic therapy, but unresolving infections even under antibiotic treatment warrants ultrasound diagnosis. Ultrasound examination of the affected breast is an appropriate initial imaging tool to look for possibilities of breast abscess or inflammatory breast disease which may have similar clinical presentations as mastitis. B-mode ultrasound typically shows nonspecific signs including skin thickening and ductal dilation without defined mass or fluid collection as seen in Figures $6 \mathrm{~A}$ and $\mathrm{B}$. A dvanced mastitis collections may present as round masses on ultrasound with surrounding hypervascularity. ${ }^{29}$ Current guidelines recommend oral antibiotic treatment with continued breastfeeding to decrease engorgement. ${ }^{30} \mathrm{Empiric}$ treatment of $\mathrm{S}$. aureus mastitis is usually with dicloxacillin or erythromycin in patients with penicillin allergies. Generally, patients will feel symptom relief after 10 days of antibiotic regimen in cases of simple mastitis. ${ }^{31}$

\section{Case \#7: Breast Abscess}

This case describes a presentation of a breast abscess. B reast abscesses commonly develop in patients with inadequately treated mastitis and continuing milk retention. ${ }^{32}$ In breastfeeding mothers, the infection most likely begins as a result of bacterial entry through a cracked nipple. Most commonly Staphylococcus is the organism, which invades through the injured area of the nipple. It begins to inhabit the nutrient rich culture breast milk. This may lead to the onset of mastitis and if it is not treated appropriately, an abscess will develop. The breast abscess contains necrotic breast tissue, denatured breast milk and pus, which are contained within the abscess. ${ }^{33}$ B reast infections are a common cause of fever in the postpartum period. M astitis occurs in 9 to $33 \%$ of lactating women. Of those with mastitis, up to $11 \%$ go on to develop a breast abscess. ${ }^{34}$

The patient presented with all the classic signs and symptoms of a serious breast infection, although, it can be difficult to differentiate a breast abscess from mastitis, especially if the abscess is small and located deep in the breast tissue. High fever and chills with malaise, with firm, tender fluctuating mass, and the presence of erythema of the areolar or periareolar area indicate abscess formation. Often the patient has a history of engorgement and cracked nipples, later followed by unilateral pain, erythema and fever. This is similar to engorgement, which can present with mild fever, but engorgement usually presents with bilateral breast pain which improves with continuous and more frequent feedings.

The work-up for a breast abscess includes $C B C$ with differential and aerobic and anaerobic cultures of the site, which can be obtained during drainage. The imaging study of choice for diagnosis is gray scale B mode and color Doppler ultrasonography of the breast. ${ }^{35}$ Generally, an abscess on ultrasound will appear as an irregular fluid-filled mass and can contain debris. ${ }^{36} \mathrm{U}$ ltrasound findings for the patient, as show in Figures 7A and B, show an ovoid parallel retroareolar hypoechoic complex cystic mass with posterior acoustic enhancement, a slightly irregular thick wall and increased flow peripherally on color flow Doppler. If diagnosis is uncertain, needle aspiration is helpful for diagnosis and to collect fluid, which can be distinguished from galactocele or even inflammatory breast cancer. ${ }^{32}$

Treatment includes analgesia, antibiotics, surgical consultation for incision and drainage or ultrasound-guided aspiration depending on the size of the abscess. Normally, breast abscesses require treatment with surgical incision and drainage under general anesthesia. Lately, treatment of breast abscesses have been accomplished by ultrasoundguided needle aspiration. In order to optimize success with needle aspiration it is found that the diameter of the abscess should be less than $3 \mathrm{~cm}$. Otherwise, catheter drainage is appropriate. ${ }^{35}$ Recommended antibiotics used for treatment 
of puerperal breast abscess are typically penicillin, such as nafcillin, oxacillin. These are effective against beta lactamase producing S. aureus. In addition, they are safe in breastfeeding mothers, because only small amounts are excreted with breast milk. $V$ ancomycin can be used in those with suspected M RSA and/or penicillin allergy.

\section{REFERENCES}

1. Y okoe DS, Christiansen CL, J ohnson R, Sandu KE, et al. Epidemiology of and Surveillance for Postpartum Infectious. Emerg Infect Dis 2001 Sep-O ct; 7(5):837-41.

2. A dair FL. The A merican Committee of $M$ aternal W elfare, Inc: The Chairman's A ddress. A m J Obstet Gynecol 1935;30:868.

3. French $L M$, Smaill FM. A ntibiotic regimens for endometritis after delivery. Cochrane Database Syst Rev 2002;(1):CD 001067.

4. Duff P. Pathophysiology and management of cesarean endometritis. Obstet Gynecol 1986;67(2):269-76.

5. Sherman D, L urie S, B etzer $M$, et al. Uterine flora at cesarean and its relationship to postpartum endometritis. O bstet $G$ ynecol 1999;94:787-91.

6. L arsen JW, H ager WD, L ivengood CH, et al. Guidelines for the diagnosis, treatment and prevention of postoperative infections. Infect Dis O bstet Gynecol 2003;11:65-70.

7. Livingston J $C$, L lata $E$, Rinehart $E$, L eidwanger $C, M$ abie $B$, Haddad B, et al. Gentamicin and clindamycin therapy in postpartum endometritis: The efficacy of daily dosing versus dosing every 8 hours. A m J Obstet Gynecol 2003;188(1): 149-52.

8. M cDermott S, Callaghan W, Szwejbka L, M ann H, Daguise V . U rinary tract infections during pregnancy and mental retardation and developmental delay. Obstet Gynecol 2000;96(1):113-19.

9. Orshan SA. M aternity, newborn and women's health nursing: Comprehensive care across the life span. Philadelphia: Lippincott Williams \& Wilkins, 2008:p760-765.

10. L eth RA, M øller JK, Thomsen RW, Uldbjerg N, N ørgaard M . Risk of selected postpartum infections after cesarean section compared with vaginal birth: A 5-year cohort study of 32,468 women. A cta Obstet Gynecol Scand 2009;88(9):976-83.

11. Cunningham G, Levano KJ, Gilstrap LC, et al. Williams obstetrics (22nd ed). New Y ork City: M cGraw-Hill; 2005: p711-724.

12. V irtanen S. U rinary infection in the puerperium. A cta O bstetricia et Gynecologica Scandinavica 1963;42:36-42.

13. Connolly A M, Thorp J M. U rinary tract infections in pregnancy. Urol Clin North A m 1999;26(1):779-87.

14. OrrettFA, Premanand N. Postpartum surveillance of bacteriuria in term vaginal deliveries. J Natl Med Assoc 1998;90(3): 177-80.

15. M CDonnold M, Friedman A, Raker C, A nderson B. Is postpartum pyelonephritis associated with the same maternal morbi dity as antepartum pyelonephritis? J M atern Fetal N eonatal M ed 2012;25(9):1709-11.

16. Ramakrishnan K, Scheid DC. Diagnosis and management of acute pyelonephritis in adults. Am Fam Physician 2005;71(5):933-42.
17. Y oo J M , et al. Diagnosing acute pyelonephritis with CT, $99 \mathrm{mT} \mathrm{C}$ DM SA SPECT, and Doppler ultrasound: A comparative study. Korean J U rol 2010;51(4):260-65.

18. Gupta K, Hooton TM, Naber KG, Wullt B, Colgan R, M iller $L G$, et al. International clinical practice guidelines for the treatment of acute uncomplicated cystitis and pyelonephritis in women: A 2010 update by the Infectious Diseases Society of A merica and the European Society for M icrobiology and Infectious Diseases. Clin Infect Dis 2011;52(5):e103-20.

19. Gardiner RA, Gwynne RA, Roberts SA. Perinephric abscess. BJ U Int 2011;107:20-23.

20. Baumgarten DA, B aumgartner BR. Imaging and radiologic management of upper urinary tract infections. U rol Clin N orth A m 1997;24(3):545-69.

21. Ifergan J, Pommier R, et al. Imaging in upper urinary tract infections. Diagn Interv Imaging 2012;93(6):509-19.

22. Jacobsen A F, Sandset PM . V enous thromboembolism associated with pregnancy and hormonal therapy. Best Pract Res Clin Haematol 2012 Sep;25(3):319-32.

23. A rya R. H ow I manage venous thromboembolism in pregnancy. Br J Haematol 2011 J un;153(6):698-708.

24. Clark P, B rennand J, Conkie JA, et al. A ctivated protein C sensitivity, protein $C$, protein $S$ and coagulation in normal pregnancy. Thromb Haemost 1998; 79:1166-70.

25. Kyrle PA, Eichinger S. Deep vein thrombosis. Lancet 2005 1;365(9465):1163-74.

26. J acobsen A F, Skjeldestad FE, Sandset PM . Incidence and risk patterns of venous thromboembolism in pregnancy and puerperium - a register-based case-control study. A m J Obstet Gynecol 2008 Feb;198(2):233.el-7.

27. M orris J M , A Igert CS, R oberts CL. Incidence and risk factors for pulmonary embolism in the postpartum period. J Thromb Haemost 2010 M ay;8(5):998-1003.

28. Committee on Health Care for U nderserved W omen, A merican College of Obstetricians and Gynecologists. A COG . Committee opinion no 361: B reastfeeding: $M$ aternal and infant aspects. Obstet Gynecol 2007;109:479-80.

29. Ferron S, A sad-Syed M, B oisserie-L acroix M, Palussière J, Hurtevent $\mathrm{G}$. Imaging benign inflammatory syndromes. Diagn Interv I maging 2012;93(2):85-94.

30. Jahanfar $\mathrm{S}, \mathrm{Ng} \mathrm{C}$, Teng $\mathrm{CL}$. A ntibiotics for mastitis in breastfeeding women. Cochrane Database Syst Rev 2009;1:CD005458.

31. Bouic-Pagès $E$, Perrochia $H$, M illet I, Taourel P. Percutaneous biopsies: Indications and techniques. Diagn Interv Imaging 2012;93(2):116-25.

32. Jatoi I. M anual of breast diseases. Philadelphia: Lippincott William and Wilkins 2002;120.

33. M asciadri N, Ferranti C. B enign breast lesions: U Itrasound. J Ultrasound 2011;14(2):55-65.

34. Branch-Elliman W, Golen TH, Gold HS, Y assa DS, Baldini LM, W right SB. Risk factors for Staphylococcus aureus postpartum breast abscess. Clin Infect Dis 2012;1;54(1):71-77.

35. U litzsch D, Nyman M K, Carlson RA. B reast abscess in lactating women: U S-guided treatment. Radiology 2004 Sep;232:904-09.

36. Ikeda D. Breast imaging: The requisites (2nd ed). St, Louis, Missouri: M osby 2010;140-41. 
1. A 28-year-old G $2 P 2$ develops a fever on the third day after an uncomplicated cesarean delivery which was performed due to arrest of descent. Her vital signs are temperature $38.6^{\circ} \mathrm{C}\left(101.4^{\circ} \mathrm{F}\right)$, pulse 88 , blood pressure $122 / 80$, respiratory rate20. On physical examination, her lungs are clear, cardiac exam is normal, abdomen is soft and nontender. There is moderate breast engorgement and moder ate uterine fundal tenderness. What is the most likely diagnosis in this patient?
a. Endometritis
b. Mastitis
c. Cystitis
d. Septic pelvic thrombophlebitis

2. A 35-year-old G $4 \mathrm{P} 4$ develops a temperature of $38.9^{\circ} \mathrm{C}$ $\left(102^{\circ} \mathrm{F}\right)$ on the third day after a cesarean delivery. She complains of foul smelling lochia. Her vital signs are temperature 102 , pulse 88 , blood pressure $122 / 80$, and respiratory rate 20 . On physical examination, her lungs are clear, cardiac exam is normal, abdomen is soft and nontender and uterine fundal tenderness is present. $W$ hat is the greatest risk factor for the patient's infection?
a. Age
b. Multiparity
c. Route of delivery
d. Multiple cervical exams

3. A 26-year-old G 1P1 delivered vaginally 28 hours ago. She had a prolonged first stage of labor, ruptured membranes for $\mathbf{2 0}$ hours and during delivery she had a 3rd-degree laceration. She received penicillin for group B Strep. She currently complains of abdominal pain and foul smelling lochia. She has a temperature of $37.9^{\circ} \mathrm{C}\left(100.3^{\circ} \mathrm{F}\right)$, pulse 77 , blood pressure $120 / 80$ and respiratory rate 18.0 ne physical exam, she has a tender uterine fundus which measures at the umbilicus. Cardio and lung exams are normal. No costovertebral angle tenderness. U rinalysis is positive for blood and negative for W BC s, leukocyte ester ase and nitrites. Urine culture is pending. What is the best treatment for this patient?
a. Vancomycin
b. Ciprofloxacin
c. TMP-SMX
d. Gentamicin and ampicillin

4. A 22-year-old G 1P1 complains of dysuria, frequency and suprapubic pain 3 days after uncomplicated vaginal delivery. She has had no previous symptoms of dysuria and is not on antibiotics. She has no fever, vaginal discharge or hematuria. She denies back pain, nausea or vomiting. O n physical exam, she is in no apparent distress, mild abdominal tenderness and has no costovertebral angle tenderness. What is next step in diagnosis?
a. Blood culture
b. CT of abdomen
c. Urinalysis
d. Pelvic exam

5. A 31-year-old G 2 P2 presents 3 weeks postpartum with complaints of fever, chills, deep breast pain and swelling, and severe fatigue. Duration of symptoms is 1 week. She has had difficulty breastfeeding due to engorgement. Her vital signs are temperature $102^{\circ} \mathrm{F}$, pulse 78 , blood pressure
120/80, respiratory rate 20. On physical examination, her lungs are clear, cardiac exam is normal, abdomen is soft and nontender. $0 \mathrm{n}$ breast examination the right breast has periareolar erythema, warmth, and a tender fluctuant mass. M oderate axillary lymphadenopathy is present. Discharge is noted with palpation of the nipple. What is most likely cause of infection and what organism is most likely responsible?
a. Fibrocystic mass; no organism associated
b. B reast abscess; $\mathrm{S}$. aureus
c. Mastitis; S. epidermidis
d. B reast engorgement; no organism associated
e. Cellulitis; S. aureus

6. A 1-year-old G 3P3 presents to the emergency department 2 days after being discharged for an uncomplicated labor and vaginal delivery. Her postpartum course has been unremarkable. Shecomplains of increasing fever, chills and malaise. She is having abdominal pain, nausea and one episode of vomiting. $\mathrm{H}$ er vital signs are temperature $38.9^{\circ} \mathrm{C}$ $\left(102{ }^{\circ} \mathrm{F}\right)$, pulse 98 , blood pressure $125 / 85$, respiratory rate 18. On examination, her lungs are clear, cardiac exam is normal, abdomen is soft, uterine fundus is firm and nontender, costovertebral angle tenderness is present. С BC reveals white blood cell count of 14,800 . Urinalysis shows $2+$ leukocyte esterase, positive nitrite and trace blood. Which of the following organisms is most likely causing her fever?
a. Escherichia coli
b. Group A Streptococcus
c. Pseudomonas
d. Chlamydia trachomatis

7. A 27-year-old G2P2 comes into clinic with dysuria for the past 7 days. She describes having fever and chills, anorexia as well. Her temperature is $38.9^{\circ} \mathrm{C}\left(102^{\circ} \mathrm{F}\right)$ and her heart rate is 80 beats/minute. Physical exam is within normal limits except for moderate left costovertebral angle tenderness. Urinalysis shows elevated leukocyte esterase and nitrites. Patient is given oral ciprofloxacin but fever does not relent over the next 4 days. What is the next step in management?
a. Ultrasound
b. Continue current ciprofloxacin regimen
c. Cystoscopy
d. Switch to IV ampicillin and gentamicin

8. A 30-year-old G1P1 is currently breastfeeding after her delivery 2 months ago. For the past weeks, she has had tenderness, erythema and induration to her left breast. Her temperature is $38.3^{\circ} \mathrm{C}\left(101^{\circ} \mathrm{F}\right)$ and she has no other signs of infection on physical exam. Ultrasound does not show presence of breast abscess. W hat is the next step in management?
a. Give antibiotics and continue feeding
b. Give antibiotics and discontinue feeding until local inflammation resolves
c. CT scan of the chest
d. No treatment

9. A 35-year-old G 2P2 is in the hospital 6 days after delivery of her second child. She began noticing right calf swelling and redness 2 days ago. She has no significant past medical history. Today, the patient develops acute shortness of breath 
and chest pain without syncope. H er blood pressure is 95/60 $\mathrm{mm} \mathrm{Hg}$ and heart rate is 110 beats per minute. Cardiac causes of chest pain have been ruled out. What is the most appropriate diagnostic study at this point?

a. Thoracic ultrasound

b. Lower extremity ultrasound

c. Spiral CT of chest

d. V entilation/perfusion lung scan

10. A 23-year-old female with past medical history of recurrent UTI sand vesicour eteral reflux as a child comes in with fever, severe chills and back pain. Her temperature is $39.5^{\circ} \mathrm{C}$ $\left(103.1^{\circ} \mathrm{F}\right)$, heart rate 100 beats per minute, and blood pressureis 110/75 mm Hg. Physical exam reveals tenderness at the right costovertebral angle. What is the most important first step in diagnosis?
a. U rinalysis
b. Transvaginal ultrasound
c. Blood culture
d. Cystoscopy

\section{Answers}

$1 \mathrm{a} ; \quad 2 \mathrm{c} ; 3 \mathrm{~d} ; 4 \mathrm{c} ; 5 \mathrm{~b} ; 6$ a; $7 \mathrm{a} ; 8 \mathrm{a} ; \quad 9 \mathrm{c} ; 10 \mathrm{a}$

\section{ABOUT THE AUTHORS}

\section{Kathrene Tajnert}

Student Physician, D epartment of M edical Education, Paul L Foster School of Medicine, Texas Tech U niversity, EI Paso, Texas, USA

\section{Wayne Tie}

Student Physician, Department of M edical Education, Paul L Foster School of M edicine, Texas Tech U niversity, EI Paso, Texas, USA

\section{Thomas J O'Neill}

Resident, Department of Radiology, Paul L Foster School of M edicine Texas Tech University, EI Paso, Texas, USA

\section{Sanja Kupesic Plavsic (Corresponding Author)}

Professor, D epartment of Obstetrics and Gynecology and Radiology Paul L Foster School of Medicine, Texas Tech University, 4800 Alberta A ve, El Paso, TX 79905, USA, Phone: +1 (915)783-1700 e-mail: sanja.kupesic@ ttuhsc.edu 